\title{
Differential expression of sodium channel mRNAs in rat peripheral nervous system and innervated tissues
}

\author{
Synnöve Beckh \\ Ruhr-Universität Bochum, Lehrstuhl für Biochemie, Universitätsstr. 150, D-4630 Bochum, and Max-Planck-Institut für \\ Biophysikalische Chemie, Am Faßberg, D-3400 Göttingen, FRG
}

Received 26 January 1990

RNA blot hybridization analyses using probes specific for sodium channels I. II and III revealed high levels of sodium channel I mRNA and low levels of sodium channel II and III mRNAs in peripheral nervous system (PNS) tissues. The developmental expression patterns of these mRNAs were generally similar to those described for the central nervous system. The small amounts of sodium channel I and III mRNAs present in tongue muscle were greatly reduced after partial denervation. Expression of the three sodium channels thus appears to be restricted to the nervous system. Putative novel additional mRNAs, specifically expressed in the PNS, were detected with a probe that recognizes nucleotide sequences common to sodium channels I, II and III.

Development; mRNA expression; Muscle; $\mathrm{Na}^{+}$channel; RNA blot hybridization analysis

\section{INTRODUCTION}

Voltage-gated sodium channels generate and propagate action potentials in excitable cells and have been electrophysiologically characterized in cells of the central (CNS) and peripheral (PNS) nervous system and other excitable tissues [1]. DNA sequences have been determined for four different sodium channels expressed in the CNS [2-4]. The distribution of sodium channels I, II [2] and III [3] has been assessed in the rat CNS by immunoprecipitation mehods [5] and by RNA blot hybridization analysis [6]. The ontogenesis of sodium channels in the CNS has been studied by neurotoxinbinding assays $[7,8]$, by immunoprecipitation methods $[5,9]$ and by RNA hybridization analysis $[6,10]$. These three sodium channels are distributed heterogeneously throughout the CNS and their expression pattern changes during development. Whether these sodium channels are expressed in tissues of the peripheral nervous system (PNS) is not clear, although immunological methods have failed to detect the respective proteins outside of the CNS [5].

Functional studies of PNS and other tissues, such as muscle and secretory glands, have provided information about the existence and character of their sodium channels [1,11-14]. RNA hybridization and immunological studies have yielded further information regarding sodium channel distribution in rat skeletal muscle [15,16] and sciatic nerve [17]. In muscle tissues,

Correspondence address: S. Beckh, Max-Planck-Institut für Biophysikalische Chemie, Abteilung 140, PO Box 2841, D-3400 Göttingen, FRG the primary structures of the rat muscle sodium channel I [18] and the rat heart sodium channel I [19] have been deduced by cloning and sequencing the respective cDNAs. Evidence for the presence of sodium channels in kidney derives from amiloride-binding studies (for review see [20]).

This study uses RNA blot hybridization analysis to examine the tissue distribution and developmental expression of sodium channel mRNAs in PNS and other rat tissues. The results indicate that rat sodium channels I, II and III are indeed expressed in the PNS. Other putative sodium channel mRNAs appear to be present in PNS tissues. The sodium channel mRNAs found in RNA from tongue nerves appear also in RNA from tongue muscle, but not denervated muscle. This suggests that these mRNAs are due to the innervation of the muscle.

\section{MATERIALS AND METHODS}

A detailed description of the preparation of CNS and PNS tissues is given in [6] and [21], respectively. Heart, skeletal muscle, tongue, uterus, stomach, small intestine, kidney, pancreas, spleen, adrenal and submandibular gland samples from the same pools of Wistar rats were prepared using methods similar to those described previously. Denervation of tongues was carried out post mortem by dissecting tongues and pulling out nerve fibers in order to separate as thoroughly as possible tongue nerves from muscle. The preparation of optic nerves consists of the complete nervus opticus cut behind the eye and shortly before its integration into the brain. Total RNA was isolated by the guanidinium thiocyanate method [22]. Each RNA sample was adjusted to a concentration of $2.5 \mu \mathrm{g} / \mu 1$, and $7.5 \mu \mathrm{g}$ of RNA was applied to each lane.

RNA blot hybridization analysis was carried out essentially as described previously [6]. The presence of equivalent amounts of ribosomal RNAs (18S and $28 \mathrm{~S}$ ) on each lane was confirmed by 
ethidium-bromide staining of the gels and their transfer to the membrane (Biodyne nylon membrane, Pall) was ascertained by methylene blue staining of the filters [23]. Sets of four separate but identically prepared RNA blots were pooled after baking and stored dry at room temperature. These were then hybridized under identical conditions with RNA probes that were simultaneously prepared using $\left[\alpha-{ }^{32}\right.$ P $]$ GTP. The RNA probes, derived from 3 '-noncoding sequences of the sodium channel I, II and III cDNAs, and from a proteincoding sequence of the sodium channel II cDNA, have been described previously [24]. The specific activities of the four probes used for hybridization were $4.1-5.5 \times 10^{9} \mathrm{dpm} / \mu \mathrm{g}$. Autoradiography was performed at $-80^{\circ} \mathrm{C}$ with an intensifying screen.

\section{RESULTS AND DISCUSSION}

\subsection{Sodium channel $I, I I$ and III $m R N A$ expression in the PNS, muscles and glands}

Total RNA was extracted from a variety of rat tissues at early and late postnatal stages of development and was subjected to RNA blot hybridization analysis using RNA probes [24] specific for sodium channel I, II and III mRNAs or a coding region probe (common probe) that recognizes the mRNAs of these three sodium channels. Results obtained with the specific probes in the different regions, along with a few CNS regions for comparison, are shown in figs 1 and 2. Hybridization signals detected in the PNS with the common probe are compared to brain signals in fig.3. The temporal patterns of mRNA expression in total brain and spinal cord, described earlier [6], could be recognized even when only one early and one late postnatal stage of development was analyzed. Therefore, postnatal day 7 (P7; P15 optic nerve is an exception) and late postnatal stages P30, P60 or P90 have been selected as reference days to monitor changes during development. In order to directly compare the relative mRNA levels of the various regions at the two different time points (fig.1), the RNAs of all these tissues were contained in the same blots and all were hybridized simultaneously.

High levels of sodium channel I mRNA were found in dorsal root ganglia and in a mixture of head nerves (fig.1A), as well as in cauda equina (not shown), a region of the lower spinal cord that is rich in nerve fibers. Lower levels of this mRNA were found in sciatic nerves and tongue nerves (see fig.2). In all these PNS tissues, as well as in spinal cord, the expression of sodium channel I mRNA was low at early postnatal stages and increased later. It appears that expression of sodium channel I mRNA in these peripheral nervous tissues follows the temporal pattern of the CNS [6], suggesting similar developmental regulation. Optic nerves and hypophysis expressed low levels of sodium channel I mRNA. Assuming similar hybridization efficiencies for the three specific probes and correcting for differences in their specific activities, sodium channel I mRNA is the most abundant of the three rat brain sodium channels in the rat PNS. This might have been expected from the high expression levels of sodium channel I mRNA [6] and protein [5] in spinal cord, whose motoneurons project directly into the PNS. However, sodium channel I protein has not yet been detected in PNS tissues [5]. This could be due to low levels of the channel protein in the membrane. The relatively high levels of sodium channel I mRNA in peripheral nerves suggests that this channel might also be expressed in glial cells, because the largest cell population in these nerves consists of Schwann cells that have voltage-gated sodium channels [13].

Muscle tissues apparently express low levels of sodium channel I mRNA, as has been reported for poly(A) ${ }^{+}$RNA from adult cardiac and skeletal muscle [24] and for total RNA from heart [18]. It was therefore not surprising that tongue muscle expressed sodium channel I mRNA in low amounts, comparable to those of heart (fig.1B). However, the mRNA levels at P7 were comparable to levels at P60 in tongue and heart, which contrasts with the usual developmental pattern observed in CNS, PNS and smooth muscle tissues. Minute amounts of sodium channel I mRNA were detected in kidney at P7, but not at P60. This mRNA was also undetectable in the glands that were examined. The finding that the levels at early postnatal stages were higher than at adult stages might indicate specific regulation of this sodium channel in these tissues or, more probably, might be caused by a dilution effect due to the relative increase of other RNAs during adulthood.

Sodium channel II mRNA levels in optic nerves and hypophysis were comparable to levels in some PNS tissues and to the level in adult spinal cord, although the P7 levels were extremely reduced when compared to the respective levels in total brain and spinal cord (fig.1A). A slight increase of this mRNA level seemed to occur during development of tissues such as dorsal root ganglia. Sodium channel II mRNA was detectable in tongue nerves (fig.2), but not in sciatic nerves. This low abundance of sodium channel II mRNA in the PNS emphasizes the predominance of sodium channel II in the CNS, suggesting that this channel might be related to synapse and network formation.

Sodium channel II mRNA was found neither in muscle nor in the other tissues studied (fig.1B). This is in agreement with results obtained in skeletal muscle [15] and heart [19] by using other sodium channel II-specific probes.

Sodium channel III mRNA levels were lower in PNS tissues than in brain and spinal cord (fig.1A). mRNA levels in optic nerves and hypophysis were comparable to levels in peripheral tissues. In all the PNS tissues studied sodium channel III mRNA levels were higher at P7 than at late postnatal stages. Thus, in the PNS, as in the CNS, sodium channel III is predominantly expressed at early postnatal stages, although maximal expression levels in the PNS are either greatly reduced or occur at significantly earlier stages. Together with the 


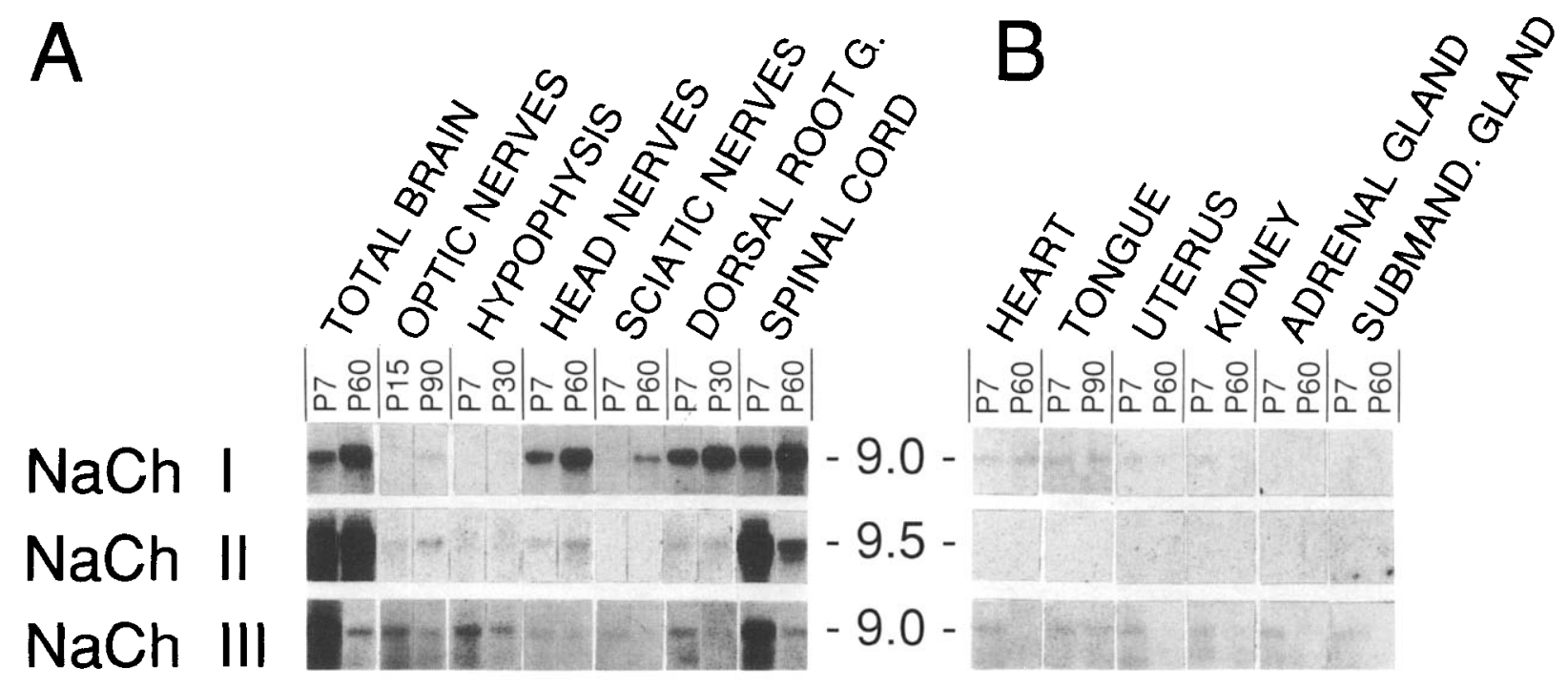

Fig.1. Autoradiograms of blot hybridization analysis of total RNA from rat tissues at early and late developmental stages. The results are presented in matrix form, showing, from top to bottom, autoradiograms of blots hybridized to the probes specific for mRNAs of sodium channel I ( $\mathrm{NaCh} \mathrm{I),} \mathrm{II} \mathrm{(} \mathrm{NaCh}$ II) and III (NaCh III), and from left to right data for the different regions at the indicated developmental stages: A, CNS and PNS tissues; B, muscle tissues, kidney and glands. The numbers after $\mathbf{P}$ indicate the postnatal age in days. The duration of autoradiography was 3 weeks. The major hybridizing mRNAs are indicated by their sizes (in kilobases, 9.0 or 9.5, respectively). Abbreviations: g., ganglia; submand., submandibular.

observed time course for sodium channel I mRNA expression, this indicates that the developmental switching of sodium channel I and III mRNAs reported for CNS development [6] is also apparent in the PNS.

Sodium channel III mRNA was detected at P7 in total RNA from a number of innervated tissues, including heart, tongue, uterus, kidney, adrenal, submandibular glands (fig.1B), and stomach and intestine (not shown). Although mRNA levels in all muscle tissues were low, the presence of some sodium channel III mRNA was expected based on previous studies of adult muscle poly(A) ${ }^{+}$RNA [24]. This mRNA, however, was hardly detectable or absent in total RNA isolated from a number of these innervated tissues at late postnatal stages. This might be a consequence of sodium channel III mRNA predominantly being expressed early in development, as seen in the nervous system. However, the low sodium channel I and III mRNA levels in these innervated tissues, and the similarity of the temporal patterns of mRNA expression between the nervous system and the innervated tissues raises the possibility that these mRNAs originate in the nerve rather than in the target tissue. This was the reason for next examining RNA from innervated and denervated tissues.

\subsection{Sodium channel I, II and III mRNA levels in partially denervated tongue muscle}

In order to distinguish between muscle-specific and nerve-specific sodium channel signals, partial denervation of muscle tissues was performed. The post mortem denervation procedure developed for this purpose (see section 2) was. chosen over the in vivo denervation techniques usually applied to skeletal muscle because it takes some time for the nerve to degenerate and because this procedure causes changes in sodium channel expression patterns $[15,19,25]$. Thus, the tongue, as a rather soft muscle which is easy to denervate by this method, was chosen for these experiments. The tongue nerves were examined separately to obtain an estimate of their contribution to the signals obtained from innervated muscle. Sodium channel I and III mRNAs were present in tongue nerves and, at much lower levels, in innervated tongue muscle (fig.2). The levels of sodium channel I and III mRNAs found in tongue muscle were greatly reduced after partial denervation. Sodium channel II mRNA was found in low levels in tongue nerves, but was undetectable in tongue or partially denervated tongue. This might reflect the low levels of this mRNA in the nerve, and the fact that nerve contributes only a small fraction of the RNA from tongue. Virtually the same results were obtained when denervation was carried out on tongues of rats at P30 and P90 (not shown). These results support the idea that the sodium channel I and III mRNAs present in tongue muscle are derived from the peripheral nerves innervating the tongue.

The mRNAs recognized by the common probe in the PNS (see fig.3) were also found in tongue nerves, but were virtually absent from tongue muscle. This suggests that these mRNAs might be nerve-specific. The two mRNA species of $\sim 9000$ and $\sim 7500$ nucleotides present in tongue muscle were only slightly reduced after partial denervation. This suggests that these mRNAs might be predominantly muscle-specific. This is consistent 


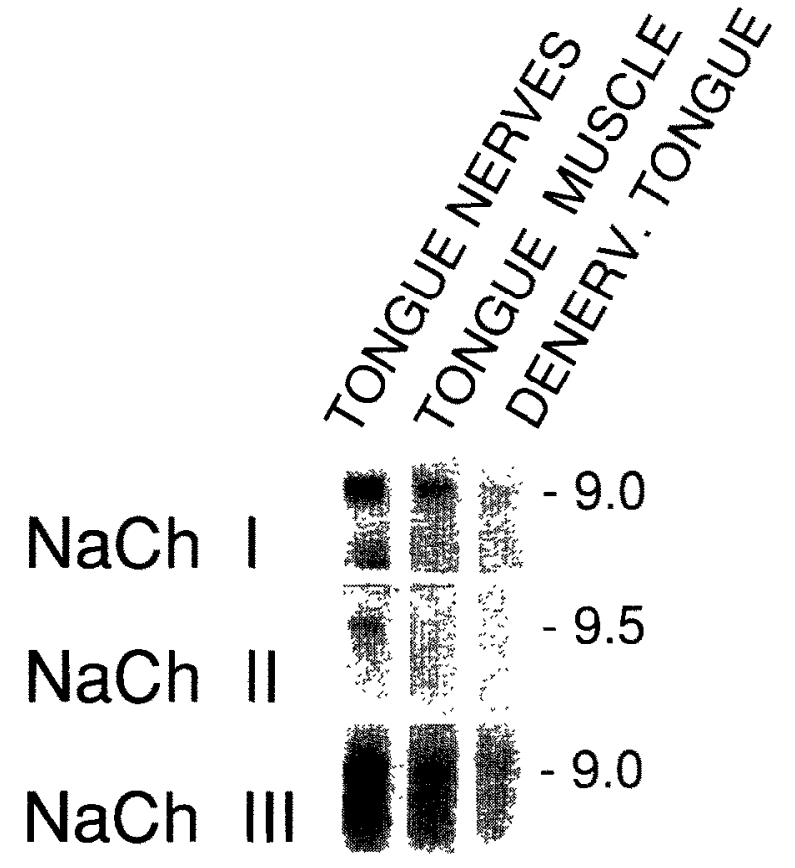

Fig.2. Autoradiograms of blot hybridization analysis of total RNA from tongue nerves, innervated and partly denervated tongues of postnatal day 7 rats. The blots were hybridized to probes specific for sodium channel I ( $\mathrm{NaCh} \mathrm{I),} \mathrm{II} \mathrm{(NaCh} \mathrm{II)} \mathrm{and} \mathrm{III} \mathrm{(} \mathrm{NaCh} \mathrm{III).} \mathrm{The}$ duration of autoradiography was 3 weeks. The major hybridizing mRNAs are indicated by their sizes (in $\mathrm{kb}$ ).

with high levels of muscle-specific mRNAs of similar sizes present in poly $(\mathrm{A})^{+}$RNA of adult skeletal muscle and heart $[15,24]$. The recently cloned rat muscle sodium channel I [18] and the putative TTX-insensitive rat heart sodium channel I [19] are encoded by transcripts of $\sim 8500$ and $\sim 9500$ nucleotides, respectively. This is comparable to the larger sized mRNA recognized by the common probe in tongue muscle. Together with the absence of the putative TTXinsensitive heart sodium channel I mRNA in innervated skeletal muscle [19], this suggests that in the innervated tongue this mRNA band consists of sodium channels I and III, muscle sodium channel I, and/or additional mRNAs of similar size. Future cloning of the respective sodium channel genes, combined with in situ hybridization, could resolve the cellular distribution of these different sodium channels.

\subsection{Additional sodium channel $m R N A s$ recognized with the common probe}

The common probe can recognize additional mRNAs encoding sodium channels in muscle (see above) and in CNS tissues [6]. In the PNS still more putative sodium channel mRNAs could be discriminated (fig.3). Besides the characterized sodium channel I $(-9000$ nucleotides), II ( $\sim 9500$ and $\sim 8600$ nucleotides), III ( $\sim 9000$ and $\sim 7500$ nucleotides) mRNAs, and the probably unspliced mRNA of $\sim 11500$ nucleotides, three

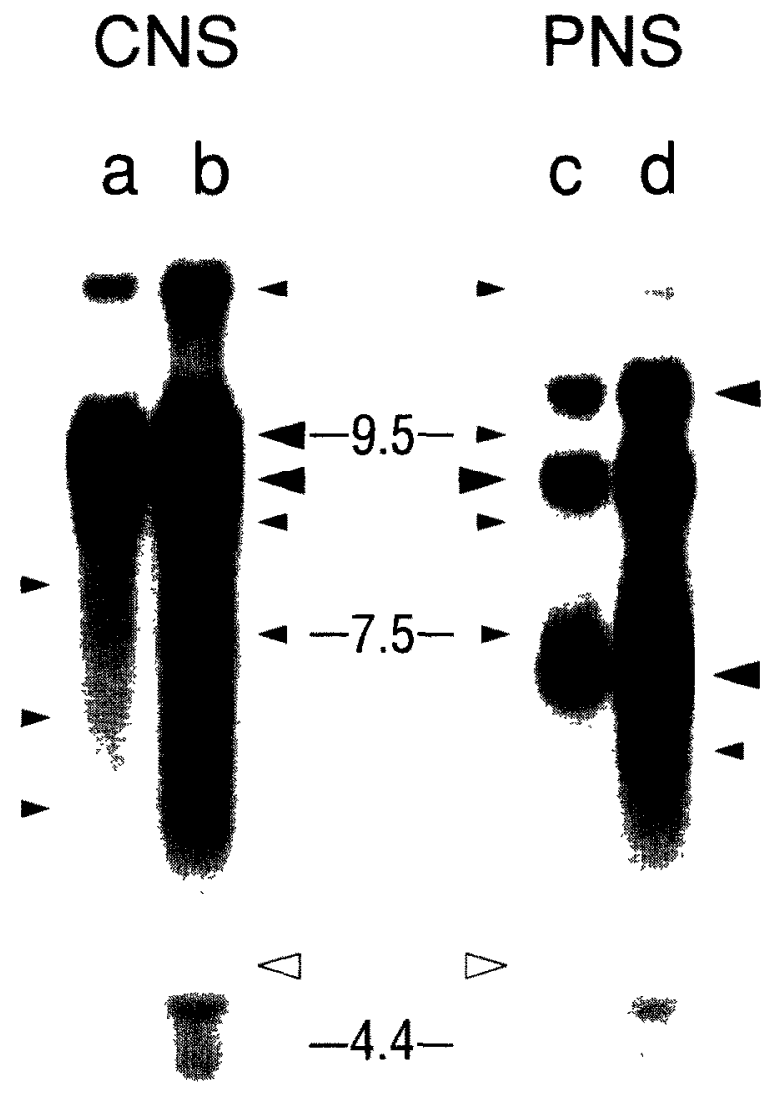

Fig.3. Autoradiograms of blot hybridization analysis of total RNA from brain (CNS) and dorsal root ganglia (PNS) of P60 rats hybridized to the common probe. Autoradiographic exposure time was $15 \mathrm{~h}$ in a and $\mathrm{c}$, and $72 \mathrm{~h}$ in $\mathrm{b}$ and $\mathrm{d}$. mRNAs corresponding to sodium channels I, II and III are indicated by arrowheads in the center of the figure. The sizes of the arrowheads reflect the relative levels of the mRNAs. Additional major and minor mRNAs are indicated by big and small arrowheads, respectively, on the outer borders of the figure. Numbers (in $\mathrm{kb}$ ) in the center indicate molecular weight markers (RNA ladder from Bethesda Research Laboratories) and open arrowheads indicate 28S rRNA.

additional mRNAs were distinguishable in the CNS and PNS. Faint mRNA bands of $\sim 8000, \sim 6700$ and $\sim 6000$ nucleotides were detected in the CNS, whereas the PNS contained two abundant mRNAs, of $\sim 10000$ and $\sim 7200$ nucleotides, and a fainter mRNA band of $\sim 6400$ nucleotides. It is possible that the shorter mRNAs detected only in the CNS or in the PNS actually are present in both, but are masked by the background smear. Because of the highly stringent conditions used and because a second, longer coding region probe revealed the same results (not shown), the strong hybridization signals are probably due to mRNAs of novel sodium channels rather than cross-hybridization to mRNAs of other ion channels.

\subsection{Distribution of $m R N A s$ recognized with the common probe}

Strong PNS-specific hybridization signals were ob- 
tained with the common probe in dorsal root ganglia (fig.3), head nerves and tongue nerves, and weaker signals were found in hypophysis and sciatic nerves (not shown). The PNS-specific mRNA of -10000 nucleotides exhibited comparable levels at early and late postnatal stages (not shown), indicating little changes during postnatal development. This mRNA could be observed during early embryonic development (at E10-E15) in RNA from whole spine that included dorsal root ganglia [6]. The $\sim 9000$ nucleotide mRNA showed increased levels in the regions studied at late postnatal stages (not shown), reflecting the temporal pattern of expression of sodium channel I mRNA. The PNS-specific mRNA of $\sim 7200$ nucleotides slightly increased during development. Undetectable levels of the two PNS-specific mRNAs in optic nerves are consistent with the CNS-related character of this nerve, whereas the presence of these mRNAs in hypophysis associates this tissue with the PNS. Sciatic nerves showed higher levels of the $\sim 7200$ nucleotide mRNA than of the other mRNA species. This and/or other mRNAs could encode voltage-gated sodium channels of glial cells $[13,26,27]$ because these nerves contain a high proportion of Schwann cells. Among the other possible proteins that could be encoded by these mRNAs are the TTX-insensitive sodium channel described in dorsal root ganglion cells [11] and a TTX-sensitive sodium channel present in fibroblasts [28].

None of the sodium channel mRNAs could be detected in liver [24], pancreas or spleen. The common probe did not hybridize to any mRNA from the glandular tissues examined or to mRNA from kidney. The absence of a sodium channel signal in kidney suggests little sequence similarity between the amiloridesensitive, voltage-independent sodium channel of kidney [20] and the voltage-gated sodium channel. In particular, this suggests that the amiloride-sensitive sodium channel does not contain an $\mathrm{S} 4$ region, which is characteristic of voltage-gated ion channels [29].

In summary, the results presented here show that sodium channels I, II and III are not only expressed in the CNS but also in the PNS. Most of the sodium channel mRNAs found in tongue muscle could be detected in tongue nerves and probably originate from the motor nerves. Therefore it is possible that sodium channels I, II and III are not present in muscle cells, although the results do not exclude the possibility that these mRNAs might be present in muscle cells in minute amounts. One conclusion of these results is that sodium channels I, II and III are only expressed in the nervous system, although sufficiently sensitive methods could detect these channels in any innervated tissue due to the presence of nerves. The putative additional sodium channel mRNAs which were detected in RNA from PNS tissues might also be present in all innervated organs, albeit in levels only detectable with specific probes or more sensitive methods.
Acknowledgements: I wish to express my gratitude to Drs Shosaku Numa, Hermann Lübbert, Peter Gruss and Erwin Neher for hospitality in their laboratories. I am grateful to Drs Petra Wahle and George Augustine for helpful comments on the manuscript. I wish to thank the Japan Society for the Promotion of Science and the Alexander von Humboldt Foundation (Feodor Lynen Program) for providing fellowships.

\section{REFERENCES}

[1] Hille, B. (1984) Ionic Channels of Excitable Membranes, Sinauer, Sunderland, MA.

[2] Noda, M., Ikeda, T., Kayano, T., Sucuki, H., Takeshima, H., Kurasaki, M., Takahashi, H. and Numa, S. (1986) Nature 320, 188-192.

[3] Kayano, T., Noda, M., Flockerzi, V., Takahashi, H. and Numa, S. (1988) FEBS Lett. 228, 187-194.

[4] Auld, V.J., Goldin, A.L., Krafte, D.S., Marshall, J., Dunn, J.M., Catterall, W.A., Lester, H.A., Davidson, N. and Dunn, R.J. (1988) Neuron 1, 449-461.

[5] Gordon, D., Merrick, D., Auld, V., Dunn, R., Goldin, A.L., Davidson, N. and Catterall, W.A. (1987) Proc. Natl. Acad. Sci. USA 84, 8682-8686.

[6] Beckh, S., Noda, M., Lübbert, H. and Numa, S. (1989) EMBO J. 8, 3611-3616.

[7] Berwald-Netter, Y., Martin-Moutot, N., Koulakoff, A. and Couraud, F. (1981) Proc. Natl. Acad. Sci. USA 78, 1245-1249.

[8] Martin-Moutot, N., Cau, P., Berwald-Netter, Y. and Couraud, F. (1987) Dev. Brain Kes. 32, 43-51.

[9] Wollner, D.A., Scheinman, R. and Catterall, W.A. (1988) Neuron 1, 727-737.

[10] Grubman, S.A., Cooperman, S.S., Begley, M.P., Weintraub, J.L., Goodman, R.H. and Mandel, G. (1988) Curr. Top. Membr. Transport 33, 277288.

[11] Ransom, B.R. and Holz, R.W. (1977) Brain Res. 136, 445-453.

[12] Ziskind-Conhaim, L. (1988) Dev. Brain Res. 42, 15-28.

[13] Chiu, S.Y. (1987) J. Physiol. 386, 181-203.

[14] Fenwick, E.M., Marty, A. and Neher, E. (1982) J. Physiol. 331, $599-635$.

[15] Cooperman, S.S., Grubman, S.A., Barchi, R.L. and Goodman, R.H. (1987) Proc. Natl. Acad. Sci. USA 84, $8721-8725$.

[16] Haimovich, B., Schotland, D.L., Fieles, W.E. and Barchi, R.L. (1987) J. Neurosci. 7, 2957-2966.

[17] Meiri, H., Steinberg, R. and Medalion, B. (1986) J. Membr. Biol. 92, 47-56.

[18] Trimmer, J.S., Cooperman, S.S., Tomiko, S.A., Zhou, I., Crean, S.M., Boyle, M.B., Kallen, R.G., Sheng, Z., Barchi, R.L., Sigworth, F.J., Goodman, R.H., Agnew, W.S. and Mandel, G. (1989) Neuron 3, 33-49.

[19] Rogart, R.B., Cribbs, L.L., Muglia, L.K., Kephart, D.D. and Kaiser, M.W. (1989) Proc. Natl. Acad. Sci. USA 86, 8170-8174.

[20] Sariban-Sohraby, S. and Benos, D.J. (1986) Am. J. Physiol. 250, C175-C187.

[21] Beckh, S. and Pongs, O. (1990) EMBO J., 9, 777-782.

[22] Chirgwin, J.M., Przybyla, A.E., MacDonald, R.J. and Rutter, W.J. (1979) Biochemistry 18, 5294-5299.

[23] Maniatis, T., Fritsch, E.F. and Sambrook, J. (1982) Molecular Cloning: A Laboratory Manual, p.206, Cold Spring Harbor Laboratory Press, Cold Spring Harbor, NY.

[24] Suzuki, H., Beckh, S., Kubo, H., Yahagi, N., Ishida, H., Kayano, T., Noda, M. and Numa, S. (1988) FEBS Lett. 228, 195-200.

[25] Weiss, R.E. and Horn, R. (1986) Science 233, 361-364. 
[26] Nowak, L., Ascher, P. and Berwald-Netter, Y. (1987) J. Neurosci. 7, 101-107.

[27] Barres, B.A., Chun, L.L.Y. and Corey, D.P. (1989) Neuron 2 , $1375-1388$.
[28] Pouysségur, J., Jacques, Y. and Lazdunski, M. (1980) Nature $286,162-164$.

[29] Stühmer, W., Conti, F., Suzuki, H., Wang, X., Noda, M. Yahagi, N., Kubo, H. and Numa, S. (1989) Nature 339, 597-603. 